\title{
DE ENEMIGO A CONCIUDADANO: EL TRÁNSITO COMO CONDICIÓN NECESARIA DEL POSTACUERDO DE LA HABANA*
}

\section{Milcíades Vizcaíno-Gutiérrez***}

Resumen: La reinserción de guerrilleros a la vida civil supone el cambio radical de la pauta cultural socializada y practicada en la guerrilla. Cinco aspectos son desarrollados como argumentos: la identidad guerrillera, la concepción de amigo-enemigo, la alianza con el campesino, la socialización de la pauta cultural y su des-socialización mediante la reintegración a la vida civil. Los argumentos se sustentan en Michel Foucault acerca del vigilar y castigar, en la construcción social de la realidad de Peter Berger y Thomas Luckmann, en las representaciones sociales de Serge Moscovici y de Denise Jodelet, y en los principios de solidaridad y de responsabilidad de Pierre Rosanvallon, el pluralismo de Chantal Mouffe y, finalmente, en la acción en la cual somos

Este artículo es parte del proyecto sobre medios, conflicto armado y Estado en Colombia que el autor desarrolla en la Universidad Cooperativa de Colombia, en la sede de Villavicencio, Departamento del Meta, Colombia.

** Sociólogo y Magíster en Educación en la especialidad de Investigación socioeducativa, investigador en la sede Villavicencio de la Universidad Cooperativa de Colombia en el Grupo de Investigación Prometeo. Docente universitario por 46 años y autor de 14 libros y numerosos artículos en revistas nacionales y extranjeras. Bogotá, Colombia. Correo-e: milciades.vizcaino@ucc.edu.co; milci.vizcaino@gmail.com. Fecha de recepción: 4 de mayo de 2017. Fecha de aceptación: 15 de enero de 2018. Para citar el artículo: MilcíAdES Vizcaíno G. "De enemigo a conciudadano: el tránsito como condición necesaria del postacuerdo de La Habana", en Revista Derecho Penal y Criminología, Vol. 38, n. ${ }^{\circ}$ 105, julio-diciembre de 2017, Bogotá, Universidad Externado de Colombia, pp. 77-99.

DOI: https://doi.org/10.18601/ 01210483.v38n105.04 
diferentes del otro, en Hannah Arendt. La información empírica proviene de documentos de las FARC y de análisis de expertos.

Palabras clave: Guerrilla de las FARC, Colombia, Socialización, Pluralismo político, Ciudadano y conciudadano.

\title{
FROM ENEMY TO FELLOW CITIZEN: TRANSIT AS A NECESSARY CONDITION OF THE POST- AGREEMENT OF HAVANA
}

\begin{abstract}
The reintegration of members of guerrilla into civilian life supposes the radical change of the cultural pause, socialized and practiced in the guerrilla. Five aspects are developed as arguments: the guerrilla identity, friendenemy conception, alliance with the peasant, socialization of cultural pause and its de-socialization through reintegration into civil life. The arguments are based on Michel Foucault's monitoring and punishing, in the social construction of reality of Peter Berger and Thomas Luckmann and in the social representations of Serge Moscovici and Denise Jodelet; in the principles of solidarity and responsibility of Pierre Rosanvallon, the pluralism of Chantal Mouffe and, finally, in action in what we are each different from the other, de Hannah Arendt. The empirical information comes from documents of the FARC and analysis from experts
\end{abstract}

Keywords: FARC Guerrilla, Colombia, Socialization, Political Pluralism, Citizens and Fellow Citizens.

\section{INTRODUCCIÓN}

El postacuerdo, postconflicto o dejación de armas significan la superación del conflicto armado de más de medio siglo de duración en Colombia. Fue la expresión de voluntad de paz de las partes en la contienda. La comunidad internacional y gran parte de los ciudadanos del país han aceptado el paso fundamental que han dado los insurgentes de las FARC con el gobierno nacional durante las negociaciones en La Habana. Los pasos acordados se cumplen en medio de dificultades porque no todo estaba dispuesto, los lugares de concentración no habían sido definidos ni contratados debidamente, las comisiones no se habían integrado totalmente, las instalaciones no se habían construido de acuerdo con las expectativas de los nuevos moradores y los contenedores no llegaron a su lugar de destino para recibir las armas en manos de los guerrilleros de acuerdo con el cronograma previsto. Estas fueron algunas de las dificultades sobresalientes. Algunos las han interpretado como deficiencias sustanciales que han estropeado el proceso y son una demostración de la improvisación y del bajo nivel de compromiso de parte del gobierno; otros, en cambio, vieron asuntos 
menores que fueron solucionados con la decisión de las partes como una demostración del cumplimiento de los acuerdos firmados.

Los hechos descritos no pueden desviar la atención ni esconder un elemento que va camuflado en medio de las actividades en la nueva cotidianidad de los insurgentes en tanto ellos se despojan de su armamento, visten de civil y hablan sobre un futuro que no ha sido vivido con anterioridad. Solamente surgen las generalidades alrededor de su participación política, tener una familia, educar a sus hijos, vivir en libertad e incorporarse a la sociedad en paz. Dentro de estos aspectos está un punto focal que consiste en la superación del concepto de enemigo que interiorizaron y anclaron en su personalidad social durante su permanencia en filas. Este elemento llama la atención en este documento y al cual se presta la atención debida porque no es contingente sino necesario para la nueva vida de los desmovilizados y para la paz en el país. De no producirse el tránsito definitivo, el riesgo de retorno a la actividad en armas está presente, como retoma de un camino aprendido durante años, ya sea en otra organización sustituta o en la delincuencia común como se desprende de experiencias similares en Centroamérica o en otros países.

El objetivo de este documento es poner en primer plano el tránsito de enemigo a ciudadano y a conciudadano. Se argumenta que si los insurgentes hacen el tránsito de una manera radical, es decir sin riesgos de retorno a las armas, se han convertido en ciudadanos que pueden vivir con un igual que es diferente, como propone Hannah Arendt ${ }^{1}$. Cuando la relación se da entre personas, sin la mediación de cosas o materia, queda al descubierto la condición humana de la pluralidad que es no solamente la conditio sine qua non de la vida política sino la conditio per quam de la vida política. Esto significa que no es una condición que precede y justifica la relación política, sino ella que es, precisamente, la esencia misma de la política como relación entre desiguales. En la política nos reconocemos entre nosotros porque somos seres humanos pero distintos en las posibilidades de pensar el ser en el mundo. Por tanto, nadie es igual a otro ni, menos aún, superior a otro. Más vigencia tiene este planteamiento en el mundo moderno en el cual las esferas social y política se diferencian mucho menos que en períodos precedentes de la historia humana.

\section{DESARROLLO}

Cinco aspectos se desarrollan como argumentos para defender la tesis planteada. Un primer aspecto es la identidad que caracteriza a la guerrilla de las FARC desde su origen hasta su lanzamiento a la vida pública, su expansión territorial y su llegada a la mesa de negociaciones. El segundo aspecto es la confrontación de dos mundos

1 ARendt, Hannah, La condición humana. Buenos Aires, Editorial Paidós, 2009. $5^{\text {a }}$ reimpresión en español. 
separados que son los de la pareja amigo-enemigo que son incompatibles por ser protagonistas de la guerra. El tercer elemento es el papel del campesino que ha sido adoptado como aliado para los guerrilleros. El cuarto aspecto es el tema de la socialización de la pauta cultural que cierra el espacio simbólico y lo hace eficaz hacia dentro e infranqueable hacia fuera. El quinto aspecto se refiere a la reintegración a la vida civil y el aprendizaje de ser y mostrase como ciudadano y conciudadano. El tratamiento de estos aspectos contribuye a dar claridad a la tesis propuesta.

\section{La identidad guerrillera}

La guerrilla ha creado para sí sus propios espacios sociales y culturales que le han permitido sobrevivir por más de cincuenta años. La travesía por montañas y llanuras, sus breves e inseguras estadías en algún punto de la geografía y los continuos desplazamientos han creado un ethos propio que les ha dado su identidad característica. Dicho ethos que crearon para sí albergó las normas y regulaciones que separaron a sus integrantes del resto de la sociedad porque establecieron barreras claramente demarcadas que no permitían el tránsito de una configuración a otra. La vida guerrillera significó la demarcación de dos regiones claramente distintas y distantes. El sociólogo Erving Goffman diferencia entre una región posterior y una región anterior $^{2}$. En el caso de las FARC, la región posterior era utilizada para desarrollar las rutinas, dar instrucciones, preparar los operativos y armarse para el combate. Esta región era, y fue, de exclusiva reserva de sus integrantes de tal suerte que no podían existir vasos comunicantes con la otra región, la región anterior, en la cual se efectuaban las operaciones como lugar de encuentro y confrontación con el enemigo. La población civil tenía limitaciones para el acceso por cuanto la clandestinidad era una de sus condiciones necesarias que aseguraban la supervivencia como organización. En otro contexto estaba el Estado con la cimentación de sus propias normas y pautas de comportamiento que rigieron para el resto de la sociedad. El resultado fue la superposición, en algunos casos, y en otros el deslinde claramente marcado por límites infranqueables en la lógica de esto o aquello, sin vasos comunicantes, en la pareja amigo-enemigo.

\section{La confrontación de amigo-enemigo}

La concepción de amigo o de enemigo establecía el arraigo indispensable o la segregación ineludible. Pero, más en profundidad, la necesidad del concepto de enemigo en la lucha guerrillera tiene una función clave para este artículo y es la anulación del campo de lo político que significa la reducción al mínimo de las diferencias y de las opciones. La diferencia con respecto al orden establecido no tiene cabida porque es el cierre de alternativas para dejar la única que es dominante y única, la que se

2 Goffman, Erving, La presentación de la persona en la vida cotidiana. Buenos Aires, Amorrortu, 1971, p. 254. 
justifica desde la causa revolucionaria. Esa causa es, en abstracto, la que justifica las determinaciones de la verticalidad del mando, la disciplina, el orden, el control e, incluso, los consejos de guerra y sus consecuencias que llegan hasta el paredón como medio de aniquilamiento de las diferencias. Todos los guerrilleros han de ser iguales en relación con el objetivo de la lucha revolucionaria; entretanto las demás variables son anuladas. En consecuencia, no hay espacio para la iniciativa, la creatividad, la opinión ni la autonomía en la decisión porque todo está regulado por otros a nombre de la causa revolucionaria. Cuando la organización, en la dirección de su jefatura autárquica, logra la "uniformidad y conformidad radical puede concebirse la imposibilidad de la lucha" en su interior que significa el fin de lo político ${ }^{3}$. Prima la voluntad del comandante y, en consecuencia, la destrucción de posibilidades de buscar alternativas en los demás integrantes de la organización que solamente son extensiones de la voluntad dominante. Para la tesis de este artículo, es el cambio más radical que se espera de los combatientes ahora desmovilizados, que asuman su papel como ciudadanos, lo que significa que puedan ejercer su vida en la confrontación de alternativas respecto de su destino colectivo en la sociedad.

El conflicto armado interno tiene como una condición esencial la confrontación en polaridades irreconciliables entre amigo-enemigo. Esto significa que hay dos campos en la organización social que son regulados por la dinámica que diferencia clara y tajantemente la vida de los guerrilleros. Sus acciones hacia dentro son de preservación de la organización y de sus vidas individuales mientras que hacia fuera es la búsqueda del enemigo para hacerle daño y para avanzar en los objetivos de la lucha insurgente. El entrenamiento militar e ideológico conlleva la clara diferenciación con las consecuencias en actitudes y en acciones que deben ser coordinadas de tal suerte que se preserve la vida y se avance en la conquista de poder para la organización. La esencia de la guerra está en la interiorización que deben hacer los guerrilleros de lo que significa el amigo tanto como el enemigo. Sin este requisito indispensable, su rol no es asimilado ni sus acciones están encaminadas a sus objetivos; se requiere un anclaje que va hasta la personalidad social de los guerrilleros. No basta la decisión de la cúpula sino que la decisión ha de irrigarse por toda la organización y alcanzar lo más profundo de cada individualidad.

El concepto de amigo-enemigo hace parte de la institucionalidad de la organización. No es posible prescindir de su contenido ni de sus alcances porque debilitaría la razón de ser de la confrontación misma. El uso de las armas significa la apelación a un medio destructor de la vida del otro, del enemigo, como objetivo central, razón de ser del conflicto. No hay términos medios, no hay espacios para la conciliación ni rendijas por las cuales se pueda filtrar un rasgo de conmiseración porque de lo que

3 Duque Muñoz, Juan Guillermo, El concepto de lo político de Carl Schmitt en movimiento: amigoenemigo y guerra. Bogotá, Universidad Colegio Mayor de Nuestra Señora del Rosario, Escuela de Ciencias Humanas, Programa de Filosofía, Monografía de grado en filosofía, 2008, p. 75. 
se trata es de la destrucción del otro para asegurar el avance en el terreno militar. En consecuencia, allí se manifiesta lo que Michel Foucault identifica como controlar la producción de los sujetos, ajustarlos al sistema social hegemónico y, de otro lado, castigar y/o sancionar las eventuales desviaciones que las circunstancias presenten ${ }^{4}$. Este aspecto merece la reflexión que evoca este documento.

La clandestinidad no permitía la visibilidad y ella no autorizaba pasar la barrera. Así se sobrevivió por 53 y más años dependiendo del criterio de demarcación original que puede estar en la crisis liberal de la mitad de la década de 1940, o el acontecimiento de la muerte del líder Jorge Eliécer Gaitán, o los decretos de amnistía del General Rojas Pinilla, o los acuerdos de Benidorm y los inicios del Frente Nacional, o el nacimiento a la vida pública de las FARC o del ELN, en 1964 ${ }^{[5]}$. Al menos tres generaciones en Colombia no conocieron otro escenario que la confrontación armada en la cual la distancia con los hechos de guerra fue, en todo caso, impredecible para nacionales y extranjeros ${ }^{6}$.

El enemigo es una construcción social que orienta la ideología de la confrontación armada y sustenta el aprendizaje necesario para todo militante de la organización. El rol guerrillero está involucrado en la tensión de los términos y de sus connotaciones. Este rol es aprendido con la incorporación y sus procesos subsiguientes hasta lograr una habituación a las expectativas creadas sobre el desempeño. No basta la subjetivación e interiorización: se requiere objetivación y, dentro de ella, la manifestación externa con actitudes y, sobre todo, acciones cuya visibilidad proporcione indicadores claros y convincentes de la correspondencia entre lo que se espera de cada uno de los integrantes del movimiento y las demostraciones en la vida cotidiana. El guerrillero está preso de su ideología pero también de la estructura de su organización representada en el mando. Desde esta perspectiva, los guerrilleros son víctimas de sí mismos. La militancia les ha construido un espacio social y cultural muy definido por normas y procedimientos que se constituyen en marcos de pensamiento y de acción. Su cotidianidad, sus operativos, sus acciones propias de sus estrategias llevan consigo marcos de interpretación que conducen decisiones y regulan su pensamiento. En consecuencia, sus percepciones, sus prácticas y sus acciones acumuladas les determinan nuevas decisiones en un engranaje envolvente que configura su propia subcultura. No solamente las estructuras narrativas o de argumentación están ligadas a esos marcos sino las estructuras mentales conscientes o inconscientes que están ligadas a los comportamientos. Los procesos cognitivos y actitudinales están involucrados claramente en la lógica de la guerra. Sin embargo, no son marcos que sean explicados en la individualidad de cada quien, sino que corresponden al conjunto de

4 Foucault, Michel, Vigilar y castigar: nacimiento de la prisión. Madrid, Siglo XXI, 1998. $27^{\text {a }}$ edición.

5 Medina Gallego, CARlos, FARC-EP y ELN Una historia política comparada (1958- 2006). Bogotá: Universidad Nacional de Colombia, Facultad de Ciencias Humanas, Departamento de Historia, 2010.

6 PÉCAUt, DANiEl, Las FARC ¿Una guerrilla sin fin o sin fines? Bogotá, Grupo Editorial Norma, 2008. 
los militantes que están unidos entre sí por la filosofía de la guerrilla, por sus concepciones sobre la vida y la muerte, por sus maneras de interiorizar la sociedad, el poder, la regulación y el orden. Este es un aspecto crucial para el proceso que viene después de la firma de los acuerdos en La Habana.

La primacía militar en las FARC supone una organización jerarquizada, con decisiones centralizadas, pero también unas prácticas de guerra irregular, subversiva, insurgente que debe responder claramente al principio marxista de la lucha de clases en la cual una de ellas ha de salir victoriosa. No es un conflicto pasajero; es la guerra en su amplio y profundo sentido, con armas y pérdida de vidas, incluso la propia como un acto revolucionario. Como refiere Alfredo Molano, "El fin de la guerra es el asesinato, dice Arteta citando a Tolstoi, y ningún guerrero que mande o cumpla órdenes -invoque la causa que invoque- puede evadir el martillazo de esta sentencia"7. Con esta premisa, queda claro el objetivo central de la guerrilla: destruir al enemigo. Este presupuesto exige que se tenga claridad sobre quién es el enemigo y en dónde está para confrontarlo hasta destruirlo. Esta es la esencia de la causa revolucionaria.

Son tres los tipos principales de enemigos que la guerrilla ha de enfrentar con las armas: uno es el macro-enemigo, del cual dependen los demás. El segundo enemigo es el imperialismo que se expresa en la política de los Estados Unidos, principalmente, como líderes de la segunda postguerra. La guerra fría dividió la geopolítica en dos bandos claramente diferenciados. Las FARC, que surgieron como autodefensas campesinas, pronto asumieron su alineación a la URSS de la cual recibieron apoyo ideológico, político y económico. Coincidían dos proyectos: uno de carácter internacional contra los Estados Unidos y su poder en el mundo, y el otro interno al país que sería la punta de lanza para la expansión en la región latinoamericana. Esta alianza delineaba una tarea que debían cumplir de cara a la historia. Las FARC mantuvieron esa posición a pesar de las transformaciones no solamente de esa federación sino de su entorno regional e internacional, y también de haber desarrollado estrategias paralelas, legitimadas políticamente, como el secuestro o la inmersión en la narcoactividad. El segundo enemigo, para los militantes de las FARC, son los defensores del imperialismo que se encuentran en cada país y que son el Estado y la clase política, pero, ante todo, los que se ubican, según ellos, en la categoría de burguesía nacional. Ellos son los conectores entre el imperialismo y el pueblo. Cumplen la doble función de congraciarse con el imperialismo y explotar al pueblo. El tercer enemigo, son los ciudadanos que hacen el juego a los dos anteriores, es decir, justifican, replican, siguen o reproducen los planteamientos del imperialismo y de la burguesía. En consecuencia, un foco de atención son los militares y policías por representar y respaldar a esa burguesía.

7 Molano, Alfredo, Presentación editorial del libro de Yesid Arteta (2014). ¡Descansen Armas! Ensayo sobre la guerra y la paz de un ex guerrillero de las FARC. Bogotá: Ícono Editorial, 2014. En la Web en http://www.iconoeditorial.com/index.html?ver=libro\&isbn=9789588461441 
Estos enemigos constituyen el blanco de las FARC. Contra ellos es que se preparan y se ejecutan acciones prioritarias que se leen en los partes de guerra que la guerrilla publicaba en su página en la Web hasta que fue neutralizada por el Estado. Los resultados, así sean pírricos, se reivindican como grandes acciones para que cumplan con la función de ley del efecto, en la concepción psicológica conductista según la cual si una respuesta guarda contigüidad con otras respuestas, en forma de cadena de impactos, tiene la posibilidad de asociarse al estímulo de tal manera que alcanza una probabilidad alta de nueva ocurrencia ante la nueva presencia del estímulo. En términos de la guerrilla: si una operación sale bien, si surte efecto, si se cumplen las premisas de su planeación, significa que esta es la vía correcta que se debe utilizar y, en consecuencia, entra a ser parte del aprendizaje colectivo. Los manuales para guerrilleros están escritos pero la realidad de la confrontación puede cambiar algunas condiciones en la ejecución que siempre deben estar sometidas a prueba. Cada acción es una demostración de que el proceso avanza, se encuentra en el mismo punto o se retrocede. En el mando centralizado de las FARC se encuentra la lectura que debe ser aceptada por la guerrillerada.

\section{El campesino es un aliado}

La guerrilla de las FARC identificó quiénes son sus enemigos y quiénes sus amigos en la confrontación. Por los orígenes de la guerrilla de las FARC, el campesinado es su aliado y la fuente de su orientación. Su lucha está enraizada y tiene sentido en el campesino. La lucha es por la reivindicación de sus intereses que están en la tierra y lo que ella produce para la vida colectiva. El centro de su plataforma política está en la tierra ${ }^{8}$. No es casual, en consecuencia, que el primer punto de la agenda de conversaciones en La Habana haya sido el problema agrario. La Reforma Rural Integral (RRI) "debe ser el inicio de transformaciones estructurales de la realidad rural y agraria de Colombia con equidad y democracia, contribuyendo así a la no repetición del conflicto y a la construcción de una paz estable y duradera", dice el borrador conjunto de los negociadores en La Habana divulgado en la Habana el 21 de junio de 2013 ,

La RRI está centrada en el bienestar y buen vivir de la gente del campo, de las comunidades campesinas, indígenas, negras, afrodescendientes, palenqueras y raizales, y de la gente que habita en los espacios interétnicos e interculturales,

8 Pizarro LeOngómez, EduARdo, "Elementos para una sociología de la guerrilla en Colombia", en Análisis Político, n. ${ }^{\circ}$ 12. Bogotá, Universidad Nacional de Colombia, Instituto de Estudios Políticos y Relaciones Internacionales (IEPRI), enero-abril, 1991, pp.4-24. PiZARro LEONGÓMEZ, EdUARDO, Insurgencia sin revolución: la guerrilla en Colombia en una perspectiva comparada. Bogotá, Tercer Mundo Editores, 1996. PiZARro LeONGómeZ, EduARdo, Las FARC (1949-2011): de guerrilla campesina a máquina de guerra. Bogotá, Grupo Editorial Norma, 2011. PiZARro LEONGómEZ, EduARdo; RiCARDO PEÑARANDA. Las FARC (1949-1966): De la autodefensa a la combinación de todas las formas de lucha. Bogotá, Tercer Mundo Editores, 1991. 
y pretende lograr la integración de las regiones, la erradicación de la pobreza, la promoción de la igualdad, el cierre de la brecha entre el campo y la ciudad, la protección y disfrute de los derechos de la ciudadanía y la reactivación del campo, especialmente de la economía campesina, familiar y comunitaria ${ }^{9}$.

Disponer de un pedazo de tierra significa la posibilidad de la vida, democratizar su uso, promover la salida de la miseria, el abandono y el conflicto sobre todo cuando la distribución se haga de manera gratuita a través del Fondo de Tierras. No es solamente una declaración sino un proyecto que en el futuro debe aplicarse; es lo que se debe hacer, como un consenso entre las partes. Complementariamente a estos beneficios, los negociadores esperan que las medidas sienten "las bases para la trasformación estructural del campo, crea condiciones de bienestar para la población rural y de esa manera contribuye a la construcción de una paz estable y duradera" ${ }^{10}$. Pero la dimensión proyectada no es solamente local ni regional porque su aplicación cobijará el territorio nacional. En el Acuerdo General para la terminación del conflicto y la construcción de una paz estable y duradera, firmado el 26 de agosto de 2012, las partes acordaron que el "desarrollo agrario integral es determinante para impulsar la integración de las regiones y el desarrollo social y económico equitativo del país" 11 .

En la segunda versión del acuerdo final, de noviembre de 2016, ratifica que el acuerdo de

"Reforma Rural Integral” contribuirá a la transformación estructural del campo, cerrando las brechas entre el campo y la ciudad y creando condiciones de bienestar y buen vivir para la población rural. La "Reforma Rural Integral" debe integrar las regiones, contribuir a erradicar la pobreza, promover la igualdad y asegurar el pleno disfrute de los derechos de la ciudadanía ${ }^{12}$.

La salida será efectiva, según el acuerdo de los negociadores, porque el territorio rural se convertirá en "un escenario socio-histórico con diversidad social y cultural” en el cual las comunidades serán sus protagonistas en el mejoramiento de sus condiciones

9 Gobierno de Colombia - FARC-EP. Primer informe conjunto de la Mesa de Conversaciones entre el Gobierno de la República de Colombia y las Fuerzas Armadas Revolucionarias de Colombia-Ejército del Pueblo, FARC-EP. 4. Descripción del acuerdo sobre el punto 1 "Política de Desarrollo Agrario Integral” de la Agenda del Acuerdo General. La Habana, 21 de junio de 2013,p. 7. Disponible en https:// www.mesadeconversaciones.com.co/comunicados/1er-informe-conjunto-mesa-de-conversacionesla-habana-21-de-junio-de-2013

10 Ídem, p.7.

11 GOBIERNO DE COLOMBIA - FARC-EP. Acuerdo General para la terminación del conflicto y la construcción de una paz estable y duradera. La Habana, 26 agosto 2012, p. 10.

12 GoBIERNO DE COLOMBIA - FARC-EP. Acuerdo final para la terminación del conflicto y la construcción de una paz estable y duradera. Bogotá, 24 noviembre, 2016, p.10. En la Web en https://www. mesadeconversaciones.com.co/sites/default/files/24-1480106030.11-1480106030.2016nuevoacuer dofinal-1480106030.pdf 
de vida y del desarrollo de todo el país ${ }^{13}$. Las expectativas de paz, en consecuencia, se juegan en el sector rural con sus protagonistas que son los campesinos y desde allí se ve el desarrollo del país.

\section{La socialización de la pauta cultural}

La pauta cultural de las FARC ha abierto espacios de relación con el Estado legítimo de Colombia. Se trata de un proceso que no es automático, no depende de la firma del documento ni de la voluntad de los negociadores de las partes, sino que pasa por el cambio de mentalidad. La mentalidad anterior a la negociación era fruto de la socialización interna que se reproducía a sí misma por fuerza de la inculcación sobre las opciones individuales. La presión de grupo, la fuerza de las instancias de la organización, el control permanente y las limitaciones que imponen las normas hacen que el marco de pensamiento y de acción se reproduzca continuamente en el ejercicio de las múltiples tareas de un guerrillero. Su desempeño eficaz le obliga a interiorizar el rol con las prácticas impuestas y legitimadas por los mandos superiores. De lo contrario se corre el riesgo de que cada quien tenga un margen de maniobra que puede atentar contra el conjunto.

Analistas como Peter Berger y Thomas Luckmann describen el proceso ontogenético de inducción amplia que experimenta un individuo de manera coherente en la organización de su mundo objetivo mediante el alcance que tiene la socialización, tanto subjetiva como objetiva. Estos autores se refieren a la tendencia fenomenológica que tenemos los seres humanos a reconocer los procesos subjetivos como realidades objetivas cuando aprehendemos la vida cotidiana como una realidad objetivada que se nos impone como algo dado, es decir, como si fuera una realidad ordenada, objetivada y ontogenizada a la cual estamos llamados a adaptarnos ${ }^{14}$. El argumento de que los objetivos de la lucha armada están por encima de cualquier decisión autónoma de individuos es contundente. No solamente es impuesta por el ejercicio de la autoridad del comandante sino a través de mecanismos de inducción que se aplican desde el momento del ingreso a la organización, sino que se requiere su ejercicio de manera permanente para asegurar la coherencia entre individuo y organización. Se trata de una organización armada ilegal que trabaja en la clandestinidad. Este hecho es fundamental para comprender el rol de guerrillero. Todo es secreto entre ellos y solamente circula entre ellos. Los límites están previstos y nada se puede filtrar hacia el exterior. Saltar las barreras, o intentar hacerlo, es castigado con la vida. La reserva de información es una estricta norma de conducta. En consecuencia, la

13 Gobierno de Colombia - FARC-EP. Primer informe conjunto de la Mesa de Conversaciones entre el Gobierno de la República de Colombia y las Fuerzas Armadas Revolucionarias de ColombiaEjército del Pueblo, FARC-EP. 4. Descripción del acuerdo sobre el punto 1 "Política de Desarrollo Agrario Integral" de la Agenda del Acuerdo General. La Habana, 21 de junio de 2013, p. 10.

14 Berger, Peter y Thomas Luckmann, La construcción social de la realidad. Buenos Aires, Amorrortu, 1998. 15 a edición en español. 
localización de un bloque, grupo o comando son parte del secreto militar, así como la preparación de operativos, el diseño de estrategias para una toma guerrillera, los desplazamientos de una base a otra, la preparación de una emboscada, la infiltración para la participación en movilizaciones agrarias o callejeras, todo está resguardado para evitar la dispersión que puede truncar los objetivos operacionales o la misma organización. La información pertinente sale del mando superior y se desgrana hacia mandos secundarios y a la base de acuerdo con el momento y la fase de la operación que es estudiada minuciosamente. El éxito de una operación consiste precisamente en tener el control sobre la información y sobre los movimientos de quienes están comprometidos en cada operación. Para los demás, es desconocido el plan y su desarrollo. Hay sistemas de compartimentación que son ejecutadas minuciosamente para dar el salto a la sorpresa y causar los efectos previstos ${ }^{15}$.

Otras operaciones tienen aparentemente menor rigor, pero son fundamentales para golpear lugares estratégicos y para infringir daños al enemigo. En este conjunto está el recaudo de impuestos de las normas 001 y 002, expedidas por las FARC en el año 2002, las contribuciones voluntarias (denominadas popularmente vacunas), el trámite en la obtención de armas, la construcción e instalación de minas antipersona, la localización del lugar, momento y estrategia para el derrame de petróleo, la voladura de una torre o de un puente, todas son operaciones que no requieren grupos numerosos de combatientes pero sí personas especializadas en las acciones militares con instrucciones exactas. La guerrilla desarrolla operaciones abiertas en espacios urbanos con militantes no uniformados que desarrollan consignas precisas en actos privados o públicos en los cuales hacen propaganda velada o encubierta de acuerdo con las circunstancias de la clandestinidad. Igual tratamiento tiene la participación en diseño de propaganda, elaboración de piezas de publicidad, escritura de documentos, preparación de artículos para ser distribuidos clandestinamente. Otro tipo de actividad es la provisión de comida, el tráfico de mercaderías y de elementos necesarios para las actividades cotidianas, como herramientas y materiales.

Nada de lo anterior puede ser realizado colectivamente si no existen mecanismos de control y de dirección, pero también unas fuertes y bien cimentadas representaciones sociales dirigidas a las actuaciones que requiere la organización en una coherencia entre la esfera individual y la personalidad social, con códigos de lenguaje clandestinos. La socialización interna activa los sistemas cognitivos requeridos por la organización. En este sentido, Serge Moscovici ${ }^{16}$ se refiere a las representaciones sociales como una modalidad particular del conocimiento, cuya función es la elaboración de los comportamientos y la comunicación entre los individuos en un corpus organizado de conocimientos y las actividades psíquicas necesarias para que la realidad física

15 López, Liliana. "Entrevista a Liliana López, alias Olga Lucía Marín, Comandante de las FARC", en LARA SAlive, PAtricia, Las mujeres en la guerra. Bogotá, Editorial Planeta, 2000, pp. 79-132.

16 Moscovici, Serge, El Psicoanálisis. Su imagen y su público. Buenos Aires, Huemul, 1979. 
y social sea asimilada por el grupo y cierre el camino de la imaginación voluntaria de los sujetos individuales. Toda representación social es, por tanto, un producto colectivo que asimilan los individuos en su vida cotidiana.

De acuerdo con la sistematización de las representaciones sociales, Castorina organiza rasgos característicos de las representaciones sociales. Ellas son:

- Una forma de conocimiento práctico derivado de experiencias de la vida cotidiana y transferida a situaciones nuevas;

- manifiestan el hecho de que un sujeto se encuentra en un grupo social a través del cual participa de la cultura y comparte la identidad social;

- son implícitas por cuanto los individuos carecen de conciencia de su existencia y del rol que cumplen en la representación compartida y tácita;

- su función consiste en reordenar los significados acerca de los elementos del mundo, modificar el sentido de los actos sociales y orientar los comportamientos;

- su activación no es permanente ni cubre la totalidad de la vida cotidiana sino solamente aquellas instancias que requieren recetas, es decir, modos de solucionar impases en las relaciones sociales;

- sustituyen a las teorías en tanto describen, clasifican y llegan a explicar la ocurrencia de acontecimientos sociales ${ }^{17}$.

Son dos enfoques, como dos aparatos teóricos distintos, los que han construido los expertos en representaciones sociales: una tendencia es el enfoque procesual, que se relaciona con el interaccionismo simbólico, y la otra es el enfoque estructural cercano teóricamente a la psicología social cognitiva ${ }^{18}$. Si se extienden los polos extremos entre los dos enfoques, el polo procesual tendría una postura socio-construccionista y el polo estructural, enfoques que se fundamentan en la aplicación del método

17 Castorina, José Antonio y Karina Viviana Kaplan, "Las representaciones sociales: problemas teóricos y desafíos educativos", en CASTORINA, JosÉ ANTONIO (comp.). Representaciones sociales. Problemas teóricos y conocimientos infantiles. Barcelona, Editorial Gedisa, 2003.

18 Fishbein, MARTin \& ICEK AJZEn. Belief, attitude, intention and behavior: An introduction to theory and research. Reading, MA: AddisonWesley, 1975. Claude Flament. "Structure, dynamique et transformation des représentations sociales”. En Abric, JEAn Claude (ed.), Pratiques Sociales et Représentations (pp. 37-57). Paris: Presses Universitaires de France, 1994. MARÍA A. BANCHS, “Aproximaciones procesuales y estructurales al estudio de las Representaciones Sociales". Papers on Social Representations, 9, 2000, pp. 3.1-3.15. JEAN Claude ABRIC. Prácticas sociales y representaciones. México, Coyoacán, 2001. CArlos José Parales-Quenza y VizCAíno-Gutiérrez, Milcíades. Las relaciones entre actitudes y representaciones sociales: elementos para una integración conceptual. Revista Latinoamericana de Psicología, vol. 39, núm. 2, 2007, pp. 351-361. 
experimental o análisis multivariados. Recientes desarrollos “proponen orientar el estudio de las representaciones sociales hacia las relaciones entre el pensamiento y el cambio social" 19 con una ubicación del sujeto en una perspectiva política e histórica, es decir, más allá del individuo como sujeto. Los dos paradigmas que fueron explicados por Deleuze y Guattari ${ }^{20}$ (1980), el de la servidumbre y el de la sujeción, suponen una actividad del sujeto hacia una construcción de la relación a sí mismo subrayando su potencial en la selección de sus acciones, sin pasividad, sin presiones ni coacciones sociales. Es una tendencia del "derecho a tener derechos", que significa la "transformación de una conciencia de sí que se vuelve más fuerte que la conciencia de las reglas y de las normas, así como de las exigencias de los sistemas dentro de los cuales se vive y se actúa"21.

Las representaciones sociales socializadas por las FARC se modifican con la inserción a la sociedad. Dejar las armas y acogerse a la vida civil significa un cambio radical de la pauta cultural que ocurre en un proceso gradualmente asumido a lo largo de las etapas que cumple la reincorporación a la sociedad.

\section{La reintegración supone el cambio de representaciones sociales}

El paso de la guerrilla a la desmovilización es complejo: no basta la entrega de las armas ni dejar de portar el uniforme y los distintivos de la organización. La adaptación a la vida civil lleva un período de tiempo que abre caminos pero no necesariamente conducen hacia un pleno ejercicio de la vida civil para todos los antiguos militantes. La experiencia en Colombia indica que la mayoría de los desmovilizados logran el cometido de incorporarse a la sociedad. El compromiso del Estado y de sus instituciones, organizaciones como la Agencia Colombiana para la Reintegración (ACR), las ONG nacionales y extranjeras creadas ad hoc, puede ayudar a facilitar los procesos de desmonte de la pareja 'amigo-enemigo' y reemplazarla por la pareja ciudadano-conciudadano como relación entre diferentes, tal como ha sido argumentado por Hannah Arendt ${ }^{22}$.

Los desmovilizados de las FARC tienen ejemplos vivientes en compañeros militantes de la organización que se acogieron a las directrices de la Agencia Colombiana para la Reintegración o a otras alternativas. De manera que no se van a enfrentar con el vacío y la improvisación. El aprendizaje alcanzado en el país es valioso para tenerlo

19 JODELET, DENISE, "El movimiento de retorno al sujeto y el enfoque de las representaciones sociales", en Cultura y Representaciones Sociales, Revista Electrónica de Ciencias Sociales, Año 3, número 5, septiembre, 2008. En la Web en http://www.culturayrs.org.mx/revista/num5/Jodelet.html

20 Deleuze, Gilles y Félix Guattari, Kafka. Para una literatura menor. México, Editorial Era, 1980.

21 Touraine, Alain Penser autrement, París: Fayard, 2007, p. 16.

22 ARENDT, HANNAH, op. cit. 
en cuenta y lograr la acogida de los nuevos desmovilizados ${ }^{23}$. En el período 2002 a 2016, la ACR ha atendido a 58.325 desmovilizados de diferentes grupos armados ilegales; solamente de las FARC han llegado al programa 16.203 personas $^{24}$. Las FARC entregaron nombres, alias y cédulas de 6.804 guerrilleros que dejan las armas en el año 2017 entran a la vida civil; falta conformar una lista de cerca de 8.000 milicianos. El compromiso del Estado es encaminar los procesos para que todos los desmovilizados directos logren los objetivos de la reintegración. Indirectamente están involucradas víctimas, otros actores civiles y militares vinculados al conflicto, funcionarios públicos, organizaciones sociales y la sociedad en general por cuanto la búsqueda de la paz es un deber compartido.

Se espera que se produzca un cambio cultural inducido desde los diferentes sectores de la sociedad en el cual la socialización de la pauta dominante de militancia o de aceptación pasiva del conflicto armado se transforme en una nueva civilidad que, a su vez, se demuestra en la reintegración a la vida civil y en el aprendizaje de ser y mostrarse como ciudadano y conciudadano. Con la desguerrillerización se deja el ser propio de la organización, que se había anclado en su personalidad social, para pasar a un nuevo ser en el que la cultura asimilada les había llevado a no hacerlo creíble por ser parte del proyecto político del Estado que la guerrilla se proponía derrocar. El Estado colombiano no será más el enemigo porque los exmilitantes ingresarán al juego político transformado en un ciudadano. El paso significa, al mismo tiempo, legitimación de un panóptico social, por cuanto serán observados por el resto de la sociedad que arrastrará memoria, estigmas, afectos y desafectos ${ }^{25}$. El proceso puede ser doloroso para quienes dejan las armas pero, en particular, para los guerrilleros de base que tienen menores defensas para enfrentar las nuevas situaciones.

En un análisis sobre la sociedad chilena, Norbert Lechner subrayaba que la "debilidad de las experiencias prácticas y los imaginarios colectivos del 'Nosotros' tiene

23 DNP (Departamento Nacional de Planeación, Consejo Nacional de Política Económica Y Social), Documento Conpes 3554. Bogotá, 01 diciembre, 2008. BARRIOs, MANUELITA, Rol y ex-rol El proceso de salida del rol paramilitar de 10 desmovilizados. Bogotá: Universidad del Rosario, Escuela de Ciencias Humanas, Monografía de grado en Sociología, 2008. LAIR, ÉRIC, Los Procesos de Desarme, Desmovilización y Reinserción de los Grupos de Autodefensa en Colombia. Una mirada cruzada sobre Centroamérica. Bogotá, enero, 2009. MEJÍA GÓMEZ, LUISA FERNANDA, La reintegración social y económica de los grupos armados ilegales en Colombia: reflexiones a partir de la trayectoria de nueve excombatientes. Bogotá: Universidad Colegio Mayor de Nuestra Señora del Rosario, Facultades de Ciencia Política y Gobierno y Relaciones Internacionales, Maestría en Estudios Políticos e Internacionales. Bogotá, 2014. ACR (Agencia Colombiana para la Reintegración). Sistema de información para la Reintegración, 2017. Cuadro n. ${ }^{\circ}$ 1. En la Web en http://www.reintegracion.gov.co/ es/la-reintegracion/Paginas/cifras.aspx

24 ACR (Agencia Colombiana para la Reintegración), Sistema de información para la Reintegración. Cuadro n. ${ }^{\circ}$ 1, 2017. En la Web en http://www.reintegracion.gov.co/es/la-reintegracion/Paginas/cifras. aspx

25 Sugerencia personal del sociólogo Jaime Amaya Monje, el 9 de abril de 2017, en correo electrónico personal. 
consecuencias para la política. A mi juicio, falta un relato que relacione los procesos políticos y las experiencias subjetivas de los ciudadanos"26. El autor menciona el gran desafío de crear un relato que otorgue sentido y valor a los esfuerzos de cambio social que deben acompañar al cumplimiento pleno de los acuerdos en La Habana. Hace 15 años, en el inicio de la primera administración del presidente Álvaro Uribe Vélez, un grupo de personas interesadas en la recomposición de Colombia, con el liderazgo de Luis Jorge Garay, hacía una radiografía en los siguientes términos:

Hay un creciente reconocimiento de la crisis genera de la sociedad colombiana, manifiesta en problemas como la exclusión social, la precariedad del Estado, la pérdida de credibilidad en la política y en los partidos políticos, la incapacidad del modelo de desarrollo para superar los problemas de pobreza y desigualdad, la fragmentación y desarticulación de la sociedad, el marcado deterioro de la convivencia ciudadana, la debilidad del sistema de justicia, seguridad y defensa, la creciente corrupción administrativa, la impunidad generalizada, las prácticas clientelistas, las múltiples violencias, la existencia y degradación del conflicto armado, el crimen organizado y el narcotráfico, que impiden la construcción colectiva de un proyecto común de nación y de democracia, que trascienda los intereses particulares de personas y grupos sociales" 27 .

Con esta sentencia contundente empieza el libro colectivo cuyas manifestaciones no han cambiado sustancialmente en 15 años. El avance consiste en que "ha venido adquiriéndose conciencia del agravamiento de la problemática social al enmarcarse en el proceso de globalización, lo que constituye un mayor desafío a la sociedad y al Estado tal como se ha construido en el país" 28 . A este escenario llegan los desmovilizados después de un arduo proceso de negociación en La Habana, entre los años 2012 y 2016, y la dejación formal de las armas el 27 de junio de 2017. Los acuerdos delinean el futuro de los guerrilleros pero también del conjunto de la sociedad "que espera su transformación y la construcción de una sociedad más justa, incluyente y democrática" 29 .

Dentro del mar de problemas persistentes, la reivindicación de lo político, en los términos de Arendt de democracia entre diferentes ${ }^{30}$, resulta de una gran prioridad no solamente por el valor en sí mismo sino por su potencial para mover otras trans-

26 LECHNER, NORBERT, La recomposición del nosotros. Un desafío cultural. Discusión de resultados del Informe de Desarrollo Humano, del 2002, del PNUD. Santiago de Chile, PNUD, 2002.

27 Garay LuIs Jorge (coord.), Repensar a Colombia. Hacia un nuevo contrato social. Bogotá, PNUD -ACCI, 2002, p. 21.

28 Ídem, Ibídem.

29 Ídem, Ibídem.

30 ARENDT, HANNAH, op. cit. 
formaciones de la sociedad partiendo de las representaciones sociales. El concepto de lo político como construcción colectiva es el fundamento de la democracia y de la orientación de los sujetos en la sociedad en un diálogo pluralista entre diversas posiciones a cambio de la imposición de una sola alternativa que se constituye en hegemónica y, en consecuencia, produce el cierre de oportunidades. El contrato de los ciudadanos con el Estado no llega ser un acto de cesión de sus derechos para esperar decisiones de su parte que anulen la esencia del sujeto que requiere expresarse con el ejercicio de sus libertades.

En este orden de ideas, la democracia ha de ser revitalizada en la traducción de principios y normas a situaciones problemáticas para los ciudadanos. Se requiere lo que Samuel Huntington ${ }^{31}$ describió como una "definición mínima” caracterizada por dos elementos claves: uno es la concepción de la democracia como "método político" para regular la fuente de autoridad, los fines de gobierno y las instituciones políticas con lo cual se reduce la carga moral y teleológica para darle una existencia real. El otro elemento es la posibilidad de verificar fácticamente la existencia o ausencia de un régimen democrático concreto para la sociedad colombiana. La característica más loable de esta "definición mínima" no es precisamente lo mínimo de la democracia sino la reducción del amplio espectro de variables a lo que es fundamental, en este caso, a la convivencia ciudadana que está, obviamente, precedida, garantizada y respaldada, por las demás condiciones de la democracia económica, social, cultural y política sin la cual la convivencia o no puede existir o tiene una existencia lánguida y de carácter meramente formal.

Por otra parte, la definición mínima de democracia se debe encontrar dentro de la institucionalidad, entre sus miembros, con las normas, las prácticas, las relaciones cotidianas, las órdenes y los procedimientos. No se puede exigir que un miembro de la institución lleve el mensaje de la democracia si no se ha formado dentro de su ambiente, si desconoce su práctica y el sentido que ella tiene para su vida personal antes que para su función en su profesión.

Un elemento adicional consiste en la superación del malestar que la democracia ha causado en los ciudadanos por una derivación incestuosa entre una definición abstracta y descontextualizada y una práctica real, cotidiana, lejana y contradictoria $^{32}$. Esta esquizofrenia entre declaraciones y hechos debe ser superada dentro de la institución y fuera de ella. Se obedece, pero no se cumple; se practica pero sin convicción. El malestar de la democracia está en la presión desde arriba de aceptar el discurso contaminado con sus insuficiencias y contradicciones que no cumplen el objetivo de desarrollar derechos sino, al contrario, producir frustraciones. Esta democracia degradada conviene a los dirigentes porque "al estado le interesan los

31 Huntington, Samuel, La tercera ola. Buenos Aires, Paidós, 1994.

32 Galli, Carlo, El malestar de la democracia. Buenos Aires, Fondo de Cultura Económica, 2013. 
individuos no los ciudadanos, porque el individuo ciudadano es crítico, informado y racional"33. Esta es una posición también argumentada por Jürgen Habermas sobre la esfera pública que está abierta a los ciudadanos para que ellos se expresen de los temas de interés general sin recibir condicionamientos ${ }^{34}$. Lo que es esencial se torna incómodo y es colocado en un lugar secundario. Por esta razón, la aplicación de la democracia en el sentido negativo, en la esquizofrenia mencionada por Carlo Galli, no es malestar en el sentido de rechazo, de estar en contra de la democracia, tampoco es malestar en la democracia como desasosiego de estar implicado en ella. Es, más bien, malestar de la democracia, de sus instituciones políticas y de su realidad social ${ }^{35}$. Es el malestar de no sentirse ciudadano, sujeto de derechos y deberes, incluido en la sociedad y respetado por sus instituciones y no relegado a una posición pasiva y acrítica con una resignación a su exclusión. El malestar se relaciona con las limitaciones de intervenir en la esfera pública como sujetos activos. No es un odio hacia la democracia ni hacia sus conciudadanos ni una hostilidad derivada de sentirse segregado y apartado sino la negación de una posibilidad en su existencia que es saberse miembro activo de un colectivo. La aplicación del criterio de concebir lo político como relaciones entre diferentes es la salida que se propone.

Cuando el ciudadano ha asimilado los mínimos de la democracia se enfrenta con lo que Ulrich Beck dice que "se nos pide que busquemos soluciones biográficas a contradicciones sistémicas: buscamos la salvación individual de problemas compartidos"36. La modernidad actual nos impulsa a la actuación individual y a hacernos atribuciones individuales de problemas y situaciones que son colectivas. Tendemos a asimilar que los únicos agentes son los individuos cuando ellos están conectados con organizaciones que los han socializado y que los respaldan en sus actuaciones a sabiendas de que las mentalidades y las representaciones sociales son construcciones colectivas que, finalmente, llegan y son asimiladas por individuos. Generalmente se produce un error de atribución que consiste en achacar a individuos lo que proviene de estructuras sobre las cuales no tiene control directo un individuo.

Si los individuos se dan cuenta de su soledad cuando deben enfrentar problemas que no son de su responsabilidad directa aparece la ausencia de comunidad, la soledad por la ausencia de los otros, que es la relación con el otro con quien pueda compartir

33 García Hernández, Yaneth Elizabeth. "Reseña de El malestar de la democracia, de Carlo Galli”, en Revista Mexicana de Análisis Político y Administración Pública. Departamento de Gestión Pública y Departamento de Estudios Políticos y de Gobierno, Volumen III, número 2, julio-diciembre, 2014, p. 356.

34 Habermas, JÜRGEN, Historia y crítica de la opinión pública. La transformación estructural de la vida pública. Barcelona, Editorial Gustavo Gili, 1994.

35 Galli, Carlo, op . cit.

36 Ulrich Beck, citado por Bauman, Zygmunt, Comunidad. En busca de seguridad en un mundo hostil. Madrid, Siglo XXI, 2003. 
decisiones vitales para ambos. En este caso, surge lo que Zygmunt Bauman escribe en su libro Comunidad,

Echamos en falta la comunidad porque echamos en falta la seguridad, una cualidad crucial para una vida feliz, pero una cualidad que el mundo que habitamos cada vez es menos capaz de ofrecer e incluso más reacio a prometer. Pero la comunidad sigue echándose en falta tenazmente, elude nuestra aprehensión o sigue desmoronándose, porque la forma en que este mundo nos incita a cumplir nuestros sueños de una vida segura no nos acerca a su cumplimiento: en vez de mitigarse, nuestra inseguridad aumenta a medida que seguimos adelante, de modo que continuamos soñando, intentándolo y fracasando" 37 .

No es fácil para los individuos involucrados, sin que haya sido su consentimiento, dentro de un sistema egoísta, individualista, responder de manera asertiva, con éxito, a las exigentes relaciones con los demás y a la convivencia social pacífica. Se presenta lo que Pierre Rosanvallon nos indica: "El gran problema de la sociedad moderna ha sido -desde su gestación-compatibilizar dos principios sociales fundamentales: por un lado el principio de solidaridad (según el cual la sociedad tiene una deuda para con sus miembros) y el principio de responsabilidad (por el cual cada individuo es dueño de su existencia y debe hacerse cargo de sí mismo)"38. En estas circunstancias es difícil para los individuos y para la colectividad discernir las responsabilidades sociales y culturales. En casos específicos, quizá sea viable la imputación ya sea a un individuo particular o a una organización. Pero frente a los nuevos riesgos, el principio de solidaridad se pone a prueba cuando pasa por la responsabilidad individual y "la noción de riesgo social pierde su carácter unificador" 39 . Pero allí no concluye la dificultad porque también se presenta una disociación entre los objetivos económicos y los objetivos sociales. Ante este dilema, sería necesario "encontrar la manera moderna de realizar cierta internalización de lo social, a fin de lograr conjuntamente modernización económica y reconstrucción del tejido social [es decir] ser moderno y arcaico al mismo tiempo" 40 . Se requiere construir equidad de oportunidades para volver a encarrilar la existencia" 41 .

Una manera de lograr una existencia encarrilada es ir a la cuestión central de la convivencia, que consiste en 'establecer la distinción 'nosotros/ellos', que es constitutiva de la política, de manera tal que sea compatible con el reconocimiento del

37 BAUMAN, ZyGMUNT, op. cit.

38 Rosanvallon, Pierre, La Nueva Cuestión Social. Repensar al Estado Providencia. Buenos Aires, Ediciones Manantial, 2011, p.21.

39 Ídem.

40 Ídem, p. 124.

41 Ídem, p. 210. 
pluralismo" 42 . Dentro de la concepción del pluralismo está el reconocimiento y la legitimación del conflicto, lo que significa diferencias e, incluso, antagonismos pero también vías legitimadas de superación de tal suerte que no se conviertan en factores de destrucción sino de construcción. Se asume que el conflicto no puede ser erradicado por estar articulado a la dinámica de la vida social y política. El problema es su control por vías reconocidas como legítimas. Los otros no pueden ser reconocidos ni tratados como enemigos y, desde luego, destruidos. Se debería aceptar que son simplemente adversarios cuyas ideas pueden ser combatidas, incluso encarnizadamente, pero no las personas que las profesan, cuyo derecho a defender esas ideas no sea cuestionado. En síntesis, no se pueden desbordar las prácticas que llevan a que el conflicto sea equivalente a antagonismo (una lucha entre enemigos) sino a un agonismo (una lucha entre adversarios) ${ }^{43}$. De esta forma, la convivencia social pacífica se preserva porque da espacio a la libertad dentro del ámbito de lo político, entendido como ciudadanía activa, deliberación sobre temas que afectan a la comunidad política, bajo el respeto de las diferencias o del otro que es diferente ${ }^{44}$. Entonces el problema no es cómo reducir la libertad sino, al contrario, cómo darle un espacio para que la autonomía individual y colectiva tenga lugar.

La existencia social ha de ser reconstruida y quizá haya que volver a Chantal Mouffe cuando plantea que "una tarea clave de la política democrática es proporcionar las instituciones que permitan que los conflictos adopten una forma agonista, donde los oponentes no sean enemigos sino adversarios entre los cuales exista un consenso conflictual" 45 . Se requiere "establecer relaciones más equitativas entre polos regionales, un enfoque multipolar podría constituir un paso hacia un orden agonista en el cual los conflictos, si bien no desaparecerían, tendrían menos probabilidades de adoptar una forma antagónica"46. La dimensión internacional de Mouffe ha de ser traducida al interior de la sociedad colombiana en su totalidad nacional, regional y local en la cual se desenvuelve la acción de individuos y organizaciones como protagonistas que deben asumir su rol preferente. A esta construcción colectiva están citados quienes han participado directa o indirectamente en el conflicto armado lo mismo que en las negociaciones entre las partes, pero también las demás instancias de la sociedad. De su articulación en la vida de los conciudadanos se verán sus resultados frente a las largas y pacientes demandas de los colombianos de poder convivir con otros diferentes en un clima de paz.

42 Mouffe, Chantal, Agonística: pensar el mundo políticamente. Buenos Aires: Fondo de Cultura Económica, 2014, p. 26.

43 Ídem.

44 Sugerencia personal del sociólogo Francisco González, candidato a PhD en New School for Social Scienses, de Nueva York.

45 Mouffe, Chantal, El retorno de lo político. Comunidad, ciudadanía, pluralismo, democracia radical. Barcelona: Paidós, 1999, p.16.

46 Mouffe, Chantal, Agonística: pensar el mundo políticamente, op. cit., p. 17. 


\section{BIBLIOGRAFÍA}

Abric, Jean Claude, Prácticas sociales y representaciones. México, Coyoacán, 2001.

ACR (Agencia Colombiana para la Reintegración), Sistema de información para la Reintegración, 2017. Cuadro n. ${ }^{\circ}$ 1. Disponible en http://www.reintegracion.gov.co/ es/la-reintegracion/Paginas/cifras .aspx

ACR (Agencia Colombiana para la Reintegración), Sistema de información para la Reintegración. Cuadro n. ${ }^{\circ}$ 1, 2017. Disponible en http://www.reintegracion.gov.co/ es/la-reintegracion/Paginas/cifras.aspx

Arendt, Hannah, La condición humana. Buenos Aires, Editorial Paidós, 2009. $5^{\text {a }}$ reimpresión en español.

BANCHS, MARía A., “Aproximaciones procesuales y estructurales al estudio de las Representaciones Sociales”, en Papers on Social Representations, 9, 2000.

Barrios, Manuelita, Rol y ex-rol El proceso de salida del rol paramilitar de 10 desmovilizados. Bogotá, Universidad del Rosario, Escuela de Ciencias Humanas, Monografía de grado en Sociología, 2008.

Bauman, Zygmunt, Comunidad. En busca de seguridad en un mundo hostil. Madrid, Siglo XXI, 2003.

Berger, Peter y Luckmann, Thomas, La construcción social de la realidad. Buenos Aires, Amorrortu, 1998. 15 a edición en español.

Castorina, José Antonio y Kaplan, Karina Viviana, "Las representaciones sociales: problemas teóricos y desafíos educativos", en José ANTONIO CASTORINA (comp.). Representaciones sociales. Problemas teóricos y conocimientos infantiles. Barcelona, Editorial Gedisa, 2003; pp. 9-27.

Deleuze, Gilles y Guattari, Félix, Kafka. Para una literatura menor. México, Editorial Era, 1980.

DNP (Departamento Nacional de Planeación, Consejo Nacional de Política Económica y Social), Documento Conpes 3554. Bogotá, 01 diciembre, 2008.

DuQue Muñoz Juan Guillermo, El concepto de lo político de Carl Schmitt en movimiento: amigo-enemigo y guerra. Bogotá, Universidad Colegio Mayor de Nuestra Señora del Rosario, Escuela de Ciencias Humanas, Programa de Filosofía, Monografía de grado en filosofía, 2008. 
Galli, CARlo, El malestar de la democracia. Buenos Aires, Fondo de Cultura Económica, 2013.

GARAy, Luis Jorge (coord.), Repensar a Colombia. Hacia un nuevo contrato social. Bogotá, PNUD -ACCI, 2002.

García Hernández, Yaneth Elizabeth, "Reseña de El malestar de la democracia, de Carlo Galli", en Revista Mexicana de Análisis Político y Administración Pública. Departamento de Gestión Pública y Departamento de Estudios Políticos y de Gobierno, Volumen III, número 2, julio-diciembre, 2014.

FISHBEIN, MARTIN \& AJZEN, ICEK, Belief, attitude, intention and behavior: An introduction to theory and research. Reading, MA: AddisonWesley, 1975.

Flament, Claude, "Structure, dynamique et transformation des représentations sociales", en Jean Claude Abric (ed.), Pratiques Sociales et Représentations (pp. 37-57). Paris, Presses Universitaires de France, 1994.

FOUCAUlt, Michel, Vigilar y castigar: nacimiento de la prisión. Madrid, Siglo XXI, 1998. $27^{a}$ edición.

Gobierno de Colombia - FARC-EP. Primer informe conjunto de la Mesa de Conversaciones entre el Gobierno de la República de Colombia y las Fuerzas Armadas Revolucionarias de Colombia-Ejército del Pueblo, FARC-EP. 4. Descripción del acuerdo sobre el punto 1 "Política de Desarrollo Agrario Integral" de la Agenda del Acuerdo General. La Habana, 21 de junio de 2013, p. 7. Disponible en https:// www.mesadeconversaciones .com.co/comunicados/1er-informe-conjunto-mesa-deconversaciones-la-habana-21-de-junio-de-2013

GOBIERNO DE COLOMBIA - FARC-EP. Acuerdo General para la terminación del conflicto y la construcción de una paz estable y duradera. La Habana, 26 agosto 2012.

GOBIERNO DE COLOMBIA - FARC-EP. Acuerdo final para la terminación del conflicto y la construcción de una paz estable y duradera. Bogotá, 24 noviembre, 2016, p.10. Disponible en https://www.mesadeconversaciones.com.co/sites/default/files/241480106030.11-1480106030.2016nuevoacuerdofinal-1480106030.pdf

Gobierno de Colombia - FARC-EP. Primer informe conjunto de la Mesa de Conversaciones entre el Gobierno de la República de Colombia y las Fuerzas Armadas Revolucionarias de Colombia-Ejército del Pueblo, FARC-EP. 4. Descripción del acuerdo sobre el punto 1 "Política de Desarrollo Agrario Integral" de la Agenda del Acuerdo General. La Habana, 21 de junio de 2013. 
GOFFMAn, ERVING, La presentación de la persona en la vida cotidiana. Buenos Aires, Amorrortu, 1971.

Habermas, Jürgen, Historia y crítica de la opinión pública. La transformación estructural de la vida pública. Barcelona, Editorial Gustavo Gili, 1994.

Huntington, Samuel, La tercera ola. Buenos Aires, Paidós, 1994.

Jodelet, Denise, "El movimiento de retorno al sujeto y el enfoque de las representaciones sociales. Cultura y Representaciones Sociales", en Revista Electrónica de Ciencias Sociales, Año 3, número 5, septiembre, 2008. Disponible en http://www. culturayrs.org.mx/revista/num5/Jodelet.html

LAIR, ÉRIC, Los Procesos de Desarme, Desmovilización y Reinserción de los Grupos de Autodefensa en Colombia. Una mirada cruzada sobre Centroamérica, Bogotá, enero, 2009.

LECHNER, NORBERT, La recomposición del nosotros. Un desafío cultural. Discusión de resultados del Informe de Desarrollo Humano, del 2002, del PNUD. Santiago de Chile, PNUD, 2002.

LóPEz, LiLIANA, “Entrevista a Liliana López, alias Olga Lucía Marín, Comandante de las FARC". En PATRICIA LARA SAlive. Las mujeres en la guerra. Bogotá, Editorial Planeta, 2000, p.79-132.

Medina Gallego, Carlos, FarC-eP y eln Una historia política comparada (19582006). Bogotá, Universidad Nacional de Colombia, Facultad de Ciencias Humanas, Departamento de Historia, 2010.

Mejía Gómez, LuISA FERnANDA, La reintegración social y económica de los grupos armados ilegales en Colombia: reflexiones a partir de la trayectoria de nueve excombatientes. Bogotá, Universidad Colegio Mayor de Nuestra Señora del Rosario, Facultades de Ciencia Política y Gobierno y Relaciones Internacionales, Maestría en Estudios Políticos e Internacionales. Bogotá, 2014.

Molano, Alfredo, Presentación editorial del libro de Yesid Arteta ;Descansen Armas! Ensayo sobre la guerra y la paz de un ex guerrillero de las FARC. Bogotá: Ícono Editorial, 2014. Disponible en http://www.iconoeditorial.com/index.html?ve $\mathrm{r}=$ libro\&isbn=9789588461441

Moscovici, Serge, El Psicoanálisis. Su imagen y su público. Buenos Aires, Huemul, 1979. 
Mouffe, Chantal, Agonística: pensar el mundo políticamente. Buenos Aires, Fondo de Cultura Económica, 2014.

Mouffe, Chantal, El retorno de lo político. Comunidad, ciudadanía, pluralismo, democracia radical. Barcelona, Paidós, 1999.

Parales-Quenza, Carlos José y Vizcaíno-Gutiérrez, Milcíades, “Las relaciones entre actitudes y representaciones sociales: elementos para una integración conceptual", en Revista Latinoamericana de Psicología, vol. 39, núm. 2, 2007, pp. 351-361.

PÉCAUT, DANIEL, Las FARC ¿Una guerrilla sin fin o sin fines? Bogotá, Grupo Editorial Norma, 2008.

Pizarro LeOngómez, EduARdo, "Elementos para una sociología de la guerrilla en Colombia". Análisis Político, n. ${ }^{\circ}$ 12. Bogotá, Universidad Nacional de Colombia, Instituto de Estudios Políticos y Relaciones Internacionales (IEPRI), enero-abril, 1991, pp. 4-24.

PIZARRo LEONGÓMEZ, EdUARDO, Insurgencia sin revolución: la guerrilla en Colombia en una perspectiva comparada. Bogotá, Tercer Mundo Editores, 1996.

Pizarro LeOngómez, EduARdo. Las FArC (1949-2011): de guerrilla campesina a máquina de guerra. Bogotá, Grupo Editorial Norma, 2011.

Pizarro Leongómez, Eduardo y Peñaranda, Ricardo, Las farc (1949-1966): De la autodefensa a la combinación de todas las formas de lucha. Bogotá, Tercer Mundo Editores, 1991.

Rosanvallon, Pierre, La Nueva Cuestión Social.Repensar al Estado Providencia. Buenos Aires, Ediciones Manantial, 2011.

Touraine, Alain, Penser autrement, París, Fayard, 2007. 